\title{
A Case-controlled Comparative Hospital-based Study on the Clinical, Biochemical, Hormonal, and Gynaecological Parameters in Polycystic Ovary Syndrome
}

\author{
MAHESWARI THANGAVELU, USHA RANI GODLA'1 , S. GODI², S. F. D. PAUL AND R. MADDALY* \\ Faculty of Biomedical Sciences, Sri Ramachandra University, ${ }^{1}$ Department of Obstetrics and Gynecology, Sri Ramachandra \\ Medical Centre, Porur, Chennai-600 116, ${ }^{2}$ Department of Human Genetics, Andhra University, Visakhapatnam-530 003, India
}

Maheswari, et al.: A Case-controlled Comparative Study on Polycystic Ovary Syndrome

\begin{abstract}
The objective of this study was to evaluate and compare the clinical, biochemical, hormonal and gynaecological aspects of polycystic ovary syndrome at a hospital in South India. The observational, case-controlled study was conducted from April 2011 to January 2014 and recruited 192 polycystic ovary syndrome patients and 205 normal women. Clinical history and biochemical and hormonal analysis were carried out. Correlation was tested between testosterone and other clinical findings. Variables were further analysed using logistic regression with adjusted odds ratio with a $95 \%$ confidence interval. About $16 \%$ of polycystic ovary syndrome women were obese and $91 \%$ reported to have an increased waist/hip ratio. Oligomenorrhea was observed in $\mathbf{7 4 \%}$ women with polycystic ovary syndrome. The tested variables revealed that body mass index, waist/hip ratio, hirsutism, testosterone, insulin, ovarian volume and follicular count were elevated in polycystic ovary syndrome patients compared to the control subjects. Increased testosterone levels correlated positively with luteinizing hormone/follicle-stimulating hormone ratio $(p=0.023)$, hirsutism $(p=0.001)$ and antral follicular count $(p=0.004)$ in polycystic ovary syndrome patients in the studied population. Waist/hip ratio could possibly be a better risk indicator than body mass index. Increased testosterone levels, ovarian volume and antral follicular count or combination of these have been considered to be risk factors in developing polycystic ovary syndrome patients.
\end{abstract}

Key words: Anovulation, hyperandrogenism, infertility, insulin resistance, hirsutism

Polycystic ovary syndrome (PCOS) is the most common and highly complex endocrinopathy, which has obtained a great deal of importance in recent years owing to it being the most prevalent cause of anovulatory infertility. Earlier, the prevalence of PCOS was found to be in approximately $7-10 \%$ of women in the reproductive age ${ }^{[1]}$. A recent, community-focused prevalence study based on Rotterdam diagnostic criteria showed an increase in the rate to $18 \%$ $(17.8 \pm 2.8 \%)^{[2]}$. In India, the prevalence ranges from $2-26 \%{ }^{[3]}$. The clinical signs and symptoms of PCOS also vary without a specific pattern among ethnic groups. This might be due to differences in lifestyle, dietary factors, genetic and biochemical make up. A common definition and definite treatment plan for PCOS does not exist, mainly due to the fact that the aetiopathogenesis of this syndrome still seems to be unclear.
According to the Rotterdam European Society for Human Reproduction/American Society of Reproductive Medicine (ESHRE/ASRM)-sponsored PCOS Consensus Group (2003) ${ }^{[3]}$, any two of the following three criteria are to be met to fit the definition of PCOS: i. Oligo- or anovulation, ii. clinical and or biochemical hyperandrogenism $(\mathrm{BH})$ and iii. polycystic ovaries on ultrasound. The most recent definition would be the guidelines proposed by Androgen Excess PCOS Society $(2006)^{[4]}$. The three diagnostic criteria for PCOS would be: i. hyperandrogenism (HA) (clinical or

This is an open access article distributed under the terms of the Creative Commons Attribution-NonCommercial-ShareAlike 3.0 License, which allows others to remix, tweak, and build upon the work non-commercially, as long as the author is credited and the new creations are licensed under the identical terms

Accepted 28 May 2017

Revised 28 January 2017

Received 26 September 2016

Indian J Pharm Sci 2017;79(4):608-616 
biochemical); ii. ovarian dysfunction (oligomenorrhea or anovulation and/or polycystic ovarian morphology) and iii. exclusion of other androgen-excess or related disorders.

PCOS is widely considered as a multi-factorial disorder, consisting of both genetic and environmental predisposing factors. The common hypothesis observed is that the genetic factors related to sex steroids result in hormonal imbalances, which predisposes a woman to HA and oligo/anovulation ${ }^{[5]}$. Other genetic factors involve genes related to obesity, genes involved in insulin action and secretion, genes involved in gonadotropin action and regulation, genes involved in ovarian and adrenal steroidogenesis. A variety of candidate genes have been postulated, however, the mode of inheritance and the exact genes implicated are not yet confirmed. PCOS manifestations include menstrual disturbances, acne vulgaris, hirsutism and most importantly, metabolic syndrome. It increases the risk of type 2 diabetes, central obesity, cardiovascular diseases, recurrent abortion, psychological and psychosexual morbidity. An inconsistency in clinical signs is observed due to its variations with age-adolescents to post-menopausal stage and other prevailing endocrinal or metabolic disorders, environmental and lifestyle changes, ethnic and geographic location. A combination of all these factors contributes to the complexity of PCOS. This study looks into the clinical, biochemical, hormonal and gynecological aspects of PCOS women visiting infertility clinic. The aim is to better understand the frequency of clinical and biochemical features of PCOS in comparison with a normal control group and to understand the underlying factors contributing to the syndrome. Also, the second objective is to see if any correlation exists between HA (testosterone levels) and other clinical manifestations of PCOS.

\section{MATERIALS AND METHODS}

\section{Study population:}

A total of 397 women were recruited from patients visiting SMART Outpatient centre and Obstetrics and Gynecology Department of Sri Ramachandra University, Chennai, Tamil Nadu, India from April 2011 to January 2014. About 192 women who fulfilled the criteria for PCOS (Rotterdam 2004) and 205 unaffected, age matched control with regular menstrual cycles (28-35 d) and normal ovaries of the same geographical area was recruited. Besides, women with galactorrhea, thyroid issues and women under medication, which interfere with the normal function of the hypothalamic-pituitary-gonadal axis or any disease, which might affect the normal reproductive physiology were excluded from the study group. This case-control study includes women in the age group of $\geq 20 \mathrm{y}$ to $\leq 40 \mathrm{y}$. The study was approved by the Institutional Ethical Committee of Sri Ramachandra University. Subject's history such as age, menarche onset, menstrual cycle regularity, marital status, consanguinity, family history with PCOS were collected after obtaining a written informed consent from all the women enrolled in the study.

\section{Sample size calculation:}

Power and sample size calculation software (version 2.1.31) was used to calculate the sample sizes in the study. We used continuity corrected chi-squared statistic to evaluate this null hypothesis. Based on power analysis, a study with 150 case patients and 150 controls was large enough to detect a significant odds ratio (OR) of 0.5 with a power of $80 \%$ and an alpha of $5 \%$.

\section{Anthropometric assessment:}

Anthropometric measurements like body height (cm), weight $(\mathrm{kg})$, hip circumference $(\mathrm{HC})$ and waist-circumference (WC, $\mathrm{cm}$ ) was assessed using a standardized instrument. In accordance to WHO classification, body-mass index (BMI) was calculated as weight in $\mathrm{kg}$ divided by height in $\mathrm{m}^{2}\left(\mathrm{~kg} / \mathrm{m}^{2}\right)$ and the women were categorized as underweight $(<18.5)$, normal (18.5-24.9), overweight (25-29.9) and obese $(\geq 30)$. WC was measured in the standing position, halfway between the lower ribs and the superior anterior iliac spine of the pelvis. $\mathrm{HC}$ was measured at the level of the pubic symphysis. The participants with waist/hip ratio (WHR) $0.80-0.84$ were classified as overweight and with WHR $>0.85$ were classified as obese.

\section{Clinical, biochemical and ultrasound assessment:}

Indication of HA was determined by presence of clinical hyperandrogenism $(\mathrm{CH})$ and/or $\mathrm{BH}$. $\mathrm{CH}$ was described by the presence of hirsutism (FerrimanGallwey (FG) score, $F G \geq 8$ ), acne, or the presence of androgenic alopecia. Hirsutism was evaluated using the modified FG scoring method. It enumerates the presence of terminal hairs over nine body areas (upper lip, chin, chest, upper and lower abdomen, upper and lower back, upper arms and thighs). Acne was recorded by the presence of comedones on the face, neck, upper chest, upper back, or upper arms. 
Biochemical HA was estimated by checking the testosterone levels in the body (total testosterone (TT) $(>76 \mathrm{ng} / \mathrm{dl}$ ) according to the normal upper limit reference value followed in the laboratory. Oligomenorrhea is defined as more than $36 \mathrm{~d}$ between menstrual cycles or fewer than eight cycles per year. Insulin resistance was assessed using the homeostatic model assessment (HOMA-IR), calculated as (fasting insulin $\times$ fasting glucose) $/ 22.5$ and $\geq 2.7^{[6,7]}$ was selected as the cut-off point. PCOS was diagnosed for the presence of 12 or more follicles in each ovary, measuring $2-9 \mathrm{~mm}$ in diameter and/or increased ovarian volume $\left(10 \mathrm{~cm}^{3}\right)$ by means of transvaginal ultrasonography with a transvaginal probe of curved array 5.0-2.0 MHz (for ovary) with a frequency of $5.9 \mathrm{MHz}$ using diagnostic ultrasound system, Sonoscape S20 Co., Ltd, China.

\section{Sample collection, biochemical and hormonal analysis:}

Peripheral blood samples were collected after an $8 \mathrm{~h}$ fast for biochemical analysis on day 2 (D2) or day 3 (D3) of their follicular phase. Thyroid profile (free $\mathrm{T} 4$, thyroid stimulating hormone (TSH), haemoglobin $(\mathrm{Hb})$, packed cell volume (PCV), pre-prandial and postprandial glucose, serum prolactin, luteinizing hormone (LH), follicle stimulating hormone (FSH), estradiol (E2), TT, fasting insulin (FI) were analysed using approved test kits, Siemens-ADVIA Centaur XP Automated System and by chemiluminescent immunoassay (CLIA).

\section{Statistical analysis:}

The characteristics between cases and controls were assessed and their percentages were calculated. Descriptive statistical analysis was performed using software SAS (version 9.0). The categorical variables estimates were analysed using Chi-square test $\left(\chi^{2}\right)$. The continuous variables were expressed as mean \pm standard deviation. The significant difference obtained in PCOS women and controls were calculated and compared using ANOVA. Correlation analysis was carried out using Pearson's correlation coefficient test. Multiple logistic regression analysis was performed with PCOS as dependent variable and various anthropometric and biochemical and hormonal variables as independent variable to find out independent risk factors for PCOS. All the variables tested for the study were included as independent variables. Totally 16 variables were included and are as follows; serum prolactin, LH, FSH, LH/FSH, estradiol, TT, insulin, HOMA-IR, menarche (y), pre and post-prandial glucose, endometrial thickness, unilateral right and left ovarian volume and unilateral right and left antral follicular count. Out of the variables tested, the variables with significant values $(\mathrm{P}<0.05)$ were represented in table along with few other variables. Thyroid profiles were retained because of the exclusion criteria of selection of subjects in the study. Also, hemoglobin and PCV were retained as those variables did not show any significant difference among PCOS and control women. The anthropometric variables were checked separately and the clinical and biochemical variables were analysed separately. We have not used any criteria for assessing model fit, as the software (SPSS) used considers default the model fit using Cox and Snell and Nagelkerke $\mathrm{R}^{2}$; hence model fit was assessed using the above said with adjusted OR and 95\% confidence interval (CI) and no interactions were examined. A P-value $<0.05$ were considered to be statistically significant.

\section{RESULTS AND DISCUSSION}

The mean age group among PCOS cases and controls were 27.69 and 26.60 , respectively. The distribution of body mass index among the subject groups were categorized as underweight, normal, overweight and obese and their respective percentages in cases and controls are $6.25,46.35,31.25,16.1$ and 5.85, 56.1, $34.63,3.42$, respectively. The distribution of $\mathrm{WC}>80$ $\mathrm{cm}$ among cases were $83 \%$ and $17 \%$ among controls. Likewise, WHR $>0.85$ among cases and controls were $91 \%$ and $9 \%$, respectively. The age onset of menarche among women ranged from $11 \mathrm{y}$ to $17 \mathrm{y}$. One hundred twenty five women and 114 women were in the 13 and 14 y age groups, respectively. Oligomenorrhea, dysmenorrhea and secondary amenorrhea among PCOS women were $74 \%, 46 \%$ and $5 \%$ while eumenorrhea was $17 \%$. Out of 160 women with irregular cycles, $5 \%$ followed $<28 \mathrm{~d}$ interval, $89 \%$ were in the $>35 \mathrm{~d}$ to $<6$-mo interval and $6 \%$ showed irregularity of having greater than or equal to 6-mo interval.

The anthropometric parameters between cases and controls are measured and presented in Table 1. Significant difference in BMI, WC, HC, WHR and hirsutism score between cases and controls was observed. The endometrial thickness, ovarian volume and follicular count among PCOS women were found to be elevated compared to that of controls. Mean hirsutism reported in control and PCOS women were 1.88 and 4.77, respectively. Table 2 represents comparison of biochemical and hormonal status between cases and controls. Except for free T4, serum prolactin, $\mathrm{Hb}$ and 
PCV, there was a significant difference between PCOS and control women.

The frequency distribution of various parameters between cases and controls are represented in
Table 3. The bilateral antral follicular count in women with PCOS was $45 \%$, the unilateral left and right being 47.4 and $50 \%$. Similarly, for the control subject's bilateral antral follicular was 3\% and the unilateral left and right count being 3.41 and 3.90\%. Also, ovarian

TABLE 1: DESCRIPTIVE ANALYSIS ON ANTHROPOMETRIC AND GYNECOLOGICAL PROFILES BETWEEN PCOS CASES AND CONTROLS

\begin{tabular}{|c|c|c|c|c|c|c|c|}
\hline \multirow{4}{*}{ Parameters } & \multicolumn{3}{|c|}{ Control } & \multicolumn{3}{|c|}{ Case } & \multirow{3}{*}{ P-value } \\
\hline & \multirow{2}{*}{ Mean } & \multicolumn{2}{|c|}{$95 \% \mathrm{Cl}$ for Mean } & \multirow{2}{*}{ Mean } & \multicolumn{2}{|c|}{$95 \% \mathrm{Cl}$ for Mean } & \\
\hline & & Lower bou & Upper bound & & Lower bou & per bound & \\
\hline & 27.69 & 27.14 & 28.23 & 26.60 & 26.00 & 27.21 & 0.009 \\
\hline Height (cm) & 155.35 & 154.52 & 156.17 & 155.44 & 154.5 & 156.37 & 0.878 \\
\hline Weight (kg) & 57.93 & 56.75 & 59.11 & 61.24 & 59.40 & 63.08 & 0.003 \\
\hline BMI & 24.01 & 23.55 & 24.47 & 25.29 & 24.61 & 25.97 & 0.002 \\
\hline Waist (cm) & 64.10 & 62.22 & 65.98 & 82.43 & 80.20 & 84.66 & 0.000 \\
\hline Hip (cm) & 86.75 & 84.78 & 88.71 & 95.98 & 93.40 & 98.56 & 0.000 \\
\hline Waist/Hip & 0.73 & 0.72 & 0.74 & 0.85 & 0.85 & 0.86 & 0.000 \\
\hline Menarche (y) & 13.83 & 13.68 & 13.99 & 13.32 & 13.16 & 13.49 & 0.000 \\
\hline Marital duration (y) & 4.86 & 4.30 & 5.41 & 4.09 & 3.67 & 4.52 & $0.033^{*}$ \\
\hline Hirsutism & 1.88 & 1.62 & 2.14 & 4.77 & 4.14 & 5.39 & 0.000 \\
\hline $\begin{array}{l}\text { Endometrial } \\
\text { thickness }\end{array}$ & 4.03 & 3.76 & 4.31 & 5.55 & 5.11 & 5.98 & $0.021^{*}$ \\
\hline $\begin{array}{l}\text { Ovarian volume } \\
\text { (right) }\end{array}$ & 4.95 & 4.43 & 5.47 & 10.44 & 9.52 & 11.36 & 0.000 \\
\hline $\begin{array}{l}\text { Ovarian volume } \\
\text { (left) }\end{array}$ & 4.36 & 3.83 & 4.88 & 9.26 & 8.57 & 9.95 & 0.000 \\
\hline AFC (Right) & 1.07 & 0.65 & 1.48 & 7.56 & 6.64 & 8.48 & 0.000 \\
\hline AFC (Left) & 0.98 & 0.59 & 1.36 & 7.32 & 6.39 & 8.25 & 0.000 \\
\hline
\end{tabular}

BMI-body mass index, AFC-antral follicular count; statistical analysis was performed using the SPSS statistical software version 9.0. The continuous variables are expressed as mean \pm standard deviation; the significant difference obtained in PCOS subjects and controls were calculated and compared using ANOVA. *P-value $<0.05$ was considered to be statistically significant

TABLE 2: DESCRIPTIVE ANALYSIS ON BIOCHEMICAL AND HORMONAL PROFILES BETWEEN PCOS CASES AND CONTROLS

\begin{tabular}{|c|c|c|c|c|c|c|c|}
\hline \multirow{3}{*}{ Parameters } & \multicolumn{3}{|c|}{ Control } & \multicolumn{3}{|c|}{ Case } & \multirow{3}{*}{ P-value } \\
\hline & \multirow[b]{2}{*}{ Mean } & \multicolumn{2}{|c|}{$95 \% \mathrm{Cl}$ for Mean } & \multirow[b]{2}{*}{ Mean } & \multicolumn{2}{|c|}{$95 \% \mathrm{Cl}$ for Mean } & \\
\hline & & $\begin{array}{l}\text { Lower } \\
\text { bound }\end{array}$ & $\begin{array}{l}\text { Upper } \\
\text { bound }\end{array}$ & & $\begin{array}{l}\text { Lower } \\
\text { bound }\end{array}$ & $\begin{array}{l}\text { Upper } \\
\text { bound }\end{array}$ & \\
\hline Free-T4 & 1.22 & 1.19 & 1.26 & 1.18 & 1.14 & 1.21 & 0.087 \\
\hline TSH & 1.9 & 1.81 & 2 & 2.41 & 2.25 & 2.57 & 0.000 \\
\hline S. Prolactin & 12.92 & 12.08 & 13.76 & 12.96 & 11.79 & 14.14 & 0.951 \\
\hline $\mathrm{LH}$ & 4.66 & 4.34 & 4.99 & 9.24 & 8.56 & 9.92 & 0.000 \\
\hline FSH & 7.05 & 6.73 & 7.38 & 6.22 & 5.92 & 6.53 & 0.000 \\
\hline LH-FSH & 0.71 & 0.65 & 0.76 & 1.63 & 1.49 & 1.76 & 0.000 \\
\hline Estradiol & 60.53 & 57.56 & 63.5 & 65.33 & 31.31 & 69.05 & $0.046^{*}$ \\
\hline $\mathrm{TT}$ & 28.7 & 26.86 & 30.53 & 71.58 & 65.33 & 77.82 & 0.000 \\
\hline Insulin & 10.66 & 10.07 & 11.26 & 13.69 & 12.63 & 14.75 & 0.000 \\
\hline HOMA-IR & 2.26 & 2.13 & 2.39 & 3.06 & 2.78 & 3.34 & 0.000 \\
\hline $\mathrm{Hb}$ & 12.87 & 12.71 & 13.04 & 13.09 & 12.87 & 13.31 & 0.118 \\
\hline PCV & 35.63 & 35.36 & 35.89 & 35.68 & 35.37 & 35.99 & 0.789 \\
\hline Pre-prandial glucose (mg/dl) & 86.04 & 84.93 & 87.16 & 89.6 & 87.18 & 92.02 & 0.008 \\
\hline Post-prandial glucose (mg/dl) & 110.49 & 108.97 & 112.01 & 116.25 & 111.45 & 121.05 & $0.021^{*}$ \\
\hline
\end{tabular}

TSH, thyroid stimulating hormone, S. prolactin- serum prolactin, LH- luteinizing hormone, FSH- follicle stimulating hormone, TT- total testosterone, HOMA-IR- homeostatic model assessment of insulin resistance, Hb- haemoglobin, PCV- packed cell volume; Statistical analysis was performed using the SPSS statistical software version 9.0. The continuous variables are expressed as mean \pm standard deviation; the significant difference obtained in PCOS subjects and controls were calculated and compared using ANOVA. *P-value $<0.05$ was considered to be statistically significant 
volume among cases and control women were 27.6 and $1.5 \%$. The overall increased testosterone level among women was $22 \%$. Among PCOS cases $24 \%$ showed hirsutism and $8 \%$ confirmed acne but no case of alopecia was observed. The primary and secondary infertility was 28.64 and $8.85 \%$ accordingly. First degree consanguinity among cases was $2.08 \%$. Second degree, third degree and distant relative consanguinity among cases and controls were 10.93, 2.60, 2.60 and $3.41 \%, 2.92,3.90 \%$ in that order. Other disorders associated include bronchial asthma (1.25\%), mumps $(5.03 \%)$ and renal disease $(0.50 \%)$. About 95\% followed mixed diet (vegetarian and non-vegetarian) while 5\% account for having a vegetarian diet.

Table 4 illustrates the correlation between testosterone and PCOS related variables for all the study participants.
Positive correlation was observed between testosterone levels and LH/FSH ratio, hirsutism and antral follicular count in PCOS women. HOMA-IR and hirsutism was negatively correlated with testosterone levels in the control group. Tables 5 and 6 determine independent predictors of risk factors for PCOS using multivariate logistic regression analysis. Independent variables tested with model fit Cox and Snell $\left(\mathrm{R}^{2} 0.657\right)$ and Nagelkerke $\left(R^{2}\right.$ 0.876). Anthropometric variables and biochemical and hormonal variables were analysed separately. Among anthropometric variables, none of the parameters showed risk but TT, ovarian volume, and antral follicular count contribute towards increased risk for PCOS.

BMI revealed quite high percentage in the PCOS group. Sixteen percent of PCOS women were obese and $31 \%$

TABLE 3: FREQUENCY DISTRIBUTION OF VARIOUS PARAMETERS IN CASES, CONTROL AND COMBINED SUBJECTS

\begin{tabular}{|c|c|c|c|}
\hline Variables & Case (\%) & Control (\%) & Total (\%) \\
\hline \multicolumn{4}{|c|}{ Polycystic ovaries ( $\geq 12$ follicles) } \\
\hline AFC (right) & $96(50)$ & $8(3.90)$ & 104(26.19) \\
\hline AFC (left) & $91(47.4)$ & $7(3.41)$ & $98(24.68)$ \\
\hline AFC (both) & $87(45.3)$ & $7(3.41)$ & $94(23.67)$ \\
\hline \multicolumn{4}{|c|}{ Ovarian volume $\left(>10 \mathrm{~cm}^{3}\right)$} \\
\hline OV (right) & 79(41.4) & 15(7.31) & $94(23.67)$ \\
\hline OV (left) & $77(40.10)$ & $6(2.92)$ & $83(20.90)$ \\
\hline OV (both) & $53(27.60)$ & $3(1.46)$ & $56(14.10)$ \\
\hline $\mathrm{PCO}(\mathrm{AFC}+\mathrm{OV})$ & $37(19.3)$ & 0 & $37(9.31)$ \\
\hline Biochemical hyperandrogenism & $87(45.31)$ & $2(0.97)$ & $89(22.41)$ \\
\hline \multicolumn{4}{|c|}{ Clinical hyperandrogenism } \\
\hline Hirsutism & $46(23.95)$ & $4(1.95)$ & $50(12.59)$ \\
\hline Acne & $16(8.33)$ & $4(1.95)$ & $20(5.03)$ \\
\hline Hirsutism+acne & $7(3.64)$ & 0 & $7(1.76)$ \\
\hline Alopecia & 0 & 0 & 0 \\
\hline Marital status overall & & & $1-18 y$ \\
\hline \multicolumn{4}{|c|}{ Infertility } \\
\hline Primary Infertility & $55(28.64)$ & 0 & $55(28.64)$ \\
\hline Secondary Infertility & $17(8.85)$ & 0 & $17(8.85)$ \\
\hline \multicolumn{4}{|c|}{ Consanguinity } \\
\hline Yes & $35(18.22)$ & $22(10.71)$ & $57(14.35)$ \\
\hline No & $154(80.20)$ & $175(85.36)$ & $329(82.87)$ \\
\hline $1^{\text {st }}$ Degree & $4(2.08)$ & 0 & $4(2.08)$ \\
\hline $2^{\text {nd }}$ Degree & $21(10.93)$ & $7(3.41)$ & $28(7.05)$ \\
\hline $3^{\text {rd }}$ Degree & $5(2.60)$ & $6(2.92)$ & $11(2.77)$ \\
\hline Distant relatives & $5(2.60)$ & $8(3.90)$ & $13(3.27)$ \\
\hline \multicolumn{4}{|c|}{ Other disorders } \\
\hline Bronchial asthma & $2(1.04)$ & $3(1.46)$ & $5(1.25)$ \\
\hline Mumps & $13(6.77)$ & $7(3.41)$ & $20(5.03)$ \\
\hline Renal disease & $1(0.52)$ & $1(0.48)$ & $2(0.50)$ \\
\hline \multicolumn{4}{|c|}{ Diet } \\
\hline Vegetarian & $7(3.64)$ & $14(6.82)$ & $21(5.28)$ \\
\hline Mixed & 185(96.3) & $191(93.1)$ & $376(94.7)$ \\
\hline
\end{tabular}

OV-ovarian volume, ET-endometrial thickness, AFC-antral follicular count, PCO- polycystic ovaries 
TABLE 4: CORRELATION ANALYSIS OF TESTOSTERONE WITH VARIABLES IN PCOS SUBJECTS

\begin{tabular}{|c|c|c|c|c|c|c|}
\hline \multirow{2}{*}{ Variables } & \multicolumn{2}{|c|}{ Cases } & \multicolumn{2}{|c|}{ Control } & \multicolumn{2}{|c|}{ Total } \\
\hline & $\mathbf{R}$ & $\mathbf{P}$ & $\mathbf{R}$ & $\mathbf{P}$ & $\mathbf{R}$ & $\mathbf{P}$ \\
\hline $\mathrm{BMI}$ & 0.059 & 0.418 & -0.023 & 0.746 & 0.121 & $0.016^{*}$ \\
\hline Waist/hip & 0.116 & 0.108 & 0.001 & 0.99 & 0.444 & 0.000 \\
\hline Serum prolactin & -0.116 & 0.109 & -0.035 & 0.62 & -0.076 & 0.128 \\
\hline $\mathrm{LH}$ & 0.082 & 0.26 & 0.059 & 0.4 & 0.346 & 0.000 \\
\hline FSH & -0.12 & 0.098 & -0.074 & 0.294 & -0.176 & 0.000 \\
\hline LH/FSH & 0.163 & $0.023^{*}$ & 0.069 & 0.322 & 0.409 & 0.000 \\
\hline HOMA-IR & -0.024 & 0.742 & -0.141 & $0.044^{*}$ & 0.111 & $0.02^{*}$ \\
\hline Hirsutism grade & 0.327 & 0.000 & -0.143 & $0.041^{*}$ & 0.425 & 0.000 \\
\hline Pre-prandial glucose & 0.049 & 0.501 & -0.017 & 0.809 & 0.107 & $0.033^{*}$ \\
\hline Post-prandial glucose & 0.08 & 0.271 & -0.034 & 0.633 & 0.121 & $0.016^{*}$ \\
\hline AFC right & 0.167 & $0.021 *$ & 0.076 & 0.28 & 0.411 & 0.000 \\
\hline AFC left & 0.206 & 0.004 & 0.086 & 0.221 & 0.433 & 0.000 \\
\hline OV right & 0.065 & 0.374 & 0.082 & 0.242 & 0.306 & 0.000 \\
\hline OV left & 0.112 & 0.124 & 0.009 & 0.903 & 0.335 & 0.000 \\
\hline ET $(\mathrm{mm})$ & 0.004 & 0.954 & -0.024 & 0.736 & 0.158 & 0.002 \\
\hline
\end{tabular}

BMI-body mass index, LH-luteinizing hormone, FSH-follicle stimulating hormone, HOMA-IR-homeostatic model assessment of insulin resistance, OV-ovarian volume, ET-endometrial thickness, AFC- antral follicular count, Correlation analysis was carried out using Pearson's correlation coefficient test. $R=-1$ to +1 ; ${ }^{*} \mathrm{P}$-value $<0.05$ was considered to be statistically significant

TABLE 5: MULTIVARIATE LOGISTIC REGRESSION ANALYSIS ON ASSOCIATION BETWEEN PCOS AND ITS ANTHROPOMETRIC VARIABLES

\begin{tabular}{|c|c|c|c|c|}
\hline \multirow[t]{2}{*}{ Variable } & \multirow{2}{*}{$\frac{\operatorname{Exp}(B)}{(\mathrm{OR})}$} & \multicolumn{2}{|c|}{$\begin{array}{c}\text { 95\% Confidence } \\
\text { Interval }\end{array}$} & \multirow[t]{2}{*}{ P-value } \\
\hline & & Lower & Upper & \\
\hline Age & 0.947 & 0.866 & 1.035 & 0.227 \\
\hline BMI & 0.788 & 0.642 & 0.966 & $0.022^{*}$ \\
\hline Waist (cm) & 0.498 & 0.498 & 1.734 & 0.93 \\
\hline $\mathrm{W} / \mathrm{H}$ ratio & 1.874 & 0.437 & 8.034 & 0.053 \\
\hline
\end{tabular}

$\mathrm{W} / \mathrm{H}$ - waist/hip ratio, the variables were analysed using multivariate logistic regression with adjusted odds ratio (OR) also with a $95 \%$ confidence interval $(\mathrm{Cl})$ and a *P-value $<0.05$ was considered to be statistically significant

TABLE 6: MULTIVARIATE LOGISTIC REGRESSION ANALYSIS ON ASSOCIATION BETWEEN PCOS AND ITS BIOCHEMICAL AND HORMONAL VARIABLES

\begin{tabular}{lcccc}
\hline \multirow{2}{*}{ Variable } & Exp (B) & $\begin{array}{c}\text { 95\% Confidence } \\
\text { interval }\end{array}$ & P-value \\
\cline { 2 - 4 } & (OR) & Lower & Upper & \\
\hline Insulin & 0.538 & 0.205 & 1.411 & 0.207 \\
LH/FSH & 3.881 & 0.231 & 65.25 & 0.346 \\
Total testosterone & 1.112 & 1.075 & 1.15 & 0.000 \\
Pre-prandial glucose & 0.811 & 0.695 & 0.947 & 0.008 \\
Post-prandial glucose & 1.024 & 0.991 & 1.058 & 0.159 \\
OV Right & 1.208 & 1.069 & 1.364 & 0.002 \\
OV Left & 1.153 & 1.029 & 1.293 & $0.014^{*}$ \\
ET (mm) & 1.194 & 0.974 & 1.464 & 0.089 \\
AFC right & 1.272 & 1.093 & 1.481 & 0.002 \\
AFC left & 1.062 & 0.919 & 1.227 & 0.413 \\
\hline
\end{tabular}

LH- luteinizing hormone, FSH- follicle stimulating hormone, OVovarian volume, ET- endometrial thickness, AFC- antral follicular count, Exp (B) - exponential (B), the variables were analysed using multivariate logistic regression with adjusted odds ratio (OR) also with a $95 \%$ confidence interval $(\mathrm{Cl})$ and a *P-value $<0.05$ was considered to be statistically significant was overweight and about $6 \%$ were lean. About $83 \%$ of the obese PCOS women showed menstrual irregularity, all of them had an increased level of insulin and $55.17 \%$ showed insulin resistance by HOMA-IR. An increase in the BMI could play a role in the alterations of the biochemical and the hormonal systems. Along with BMI, waist circumference and waist/hip ratio was calculated, which are the measures of visceral or abdominal fat mass. These measures are independent of height and muscle mass. They have begun as important predictors of risk of obesity related diseases and hence are thought to be very useful indicators of excess body fat and increased health risk $^{[8]}$. About $83 \%$ PCOS women and 17\% normal women had a waist circumference of $>80 \mathrm{~cm}$, which is all of obese women had an increased waist circumference. A study reported both lean and obese PCOS women showing higher waist circumference ${ }^{[9]}$. Likewise, an increase in waist/hip ratio of about $91 \%$ was seen in PCOS group while control women had a much lower percentage of $9 \%$. Out of 181 women with increased waist/hip ratio, insulin resistance and irregular cycles was observed in $47.5 \%$ and $82 \%$ women with PCOS. Obesity prevalence was reported by Carmina et al. and Targher et al. with 15.8 and 14.3 in Italy, 13.5 in Australia and $12.5 \%$ in Israel $^{[10-13]}$. Prevalence of obesity as high as $94.6,62.8$ and $60.1 \%$ in USA, 59.7 in Bahrain, 40 in Spain and $50 \%$ in UK has also been reported. A prevalence of overweight and obesity percentage similar to our study was reported in Italy, Taiwan, Germany and Greece with $46.4,41.4,43.8$ and $47 \%$ correspondingly ${ }^{[14]}$. 
Higher waist circumference in lean and obese PCOS women was observed ${ }^{[15]}$.

All the control women in the study satisfied the criteria of having regular menstrual cycles and the same was also observed among 17\% PCOS women; irregular menstrual cycles prevail high about $83 \%$ amongst PCOS women. Among PCOS women, $74 \%$ were oligomenorrhic, $23 \%$ had a heavy menstrual flow and $12 \%$ underwent scanty flow and about 5\% women suffered from secondary amenorrhea. Any disturbances in the menstrual cycle in turn disturb the hormonal mechanisms and add to female infertility issues. Studies have reported women with oligomenorrhea as $10.2,64.3,71$ and $93 \%^{[16-19]}$. In the present study, LH was found to be elevated compared to FSH in PCOS women, which was twice as high compared to the control group due to increase in GnRH pulse frequency in PCOS women ${ }^{[20]}$. The LH/FSH ratio in our study was $31 \%$. The increase in LH levels could be due to high and static concentrations of oestrogens that alter the control of this hormone by the H-P axis ${ }^{[21]}$. Increased GnRH secretion drives the preovulatory LH surge in a dose-dependent fashion. During the follicular phase in parallel with increasing oestradiol concentrations $\mathrm{LH}$ responses to identical $\mathrm{GnRH}$ stimuli. Increased oestradiol also may act to augment the gonadotrope's responsiveness to $\mathrm{GnRH}^{[22]}$. During the normal menstrual cycle, central and pituitary sites respond to the oestradiol positive feedback loop signal to ensure a timely gonadotropin surge. Also, a significant difference was observed in the testosterone levels of PCOS women compared to that of controls, corroborating with the hypothesis that an increase in testosterone levels leads to suppression of normal menstruation and ovulation confirming with our results in the multivariate logistic regression that it withholds risk factor for PCOS.

The augmented GnRH pulse frequency is vastly associated with HA and increased ovarian volume ${ }^{[23]}$. In addition, an increase in the FI levels in the case group when compared to the control group led to the calculation of insulin resistance using HOMA-IR. The mean HOMA-IR of PCOS and control women was 3.06 and 2.26, respectively. Most women affected with PCOS in the study group did not have family members affected with the same except for a few with the proband's siblings and maternal aunts were positive for PCOS. Consanguinity was seen among the study groups with higher second degree consanguineous marriages among case group (11\%) compared to the control group
$(3 \%)$. Out of all the other variables, which impact PCOS, an elevated TT level was found in $45 \%$ women in the case group. Relationship between testosterone and other baseline parameters were assessed to find a correlation. We found that the hirsutism grades were positively correlated significantly among the case group $(\mathrm{R}=0.327 ; \mathrm{P}=0.000)$ and the combined group $(\mathrm{R}=0.425 ; \mathrm{P}=0.000)$, which ratifies that a rise in testosterone levels above a normal range leads to an unusual increase in hair growth (hirsutism). About 24\% PCOS women were hirsute and $8 \%$ showed symptoms of acne. An increased conversion of testosterone to dihydrotestosterone via the enzyme 5 alpha reductase $(5 \alpha-\mathrm{R})$ within the pilosebaceous unit may be one of the root causes for hirsutism ${ }^{[24]}$. 5- $\alpha$ reductase acts as an amplifier of the androgen signal ${ }^{[25]}$. There are two isoenzymes of 5- $\alpha$ reductase, which is type 1 and type 2 ( $5 \alpha-R$ type 1 and $5 \alpha-R$ type 2 ), characterized by studies of molecular cloning ${ }^{[26,27]}$. The predominant being $5 \alpha-\mathrm{R}$ type 1 (SDR5A1) is found majorly in $\operatorname{skin}^{[28]}$. A rise in 5a-reductase activity was seen in genital and pubic skin fibroblasts from hirsute patients compared to the skin of normal women ${ }^{[29,30]}$.

In PCOS women who are positive for HA, 11.49\% women were obese, $95.40 \%$ showed increased waist/ hip ratio, increased LH/FSH was found in $43.67 \%$, HOMA-IR was seen in $50.57 \%$, hirsutism was seen in $40.22 \%$ and infertility was observed in $44 \%$ women. A rise in testosterone levels correlates with increase in LH/FSH ratio, antral follicular count and hirsutism. PCOS women showed increased levels of insulin. High basal insulin levels (35.7\%) were reported in a study carried out with seventy women with PCOS in the age range 12-35 y of age ${ }^{[31]}$. Increased concentration of insulin binds to insulin growth factor 1 (IGF-1) receptor of theca cells leading to increased levels of androstenedione and $\mathrm{HA}^{[32]}$. Earlier studies have shown that combination of LH/FSH ratio and FI level were of higher predictive value in diagnosing PCOS ${ }^{[33]}$. Too much insulin combined with high levels of LH can lead to excess production of testosterone in the ovaries. These abnormally high levels of testosterone in turn lead to disruption in the follicle maturation process, which hampers ovulation ${ }^{[34-36]}$. Excess androgens boost insulin resistance leading to raised insulin levels, which in turn fuel androgen synthesis. This increases the symptoms of PCOS, making women more susceptible to diabetes, obesity and cardiovascular ailments ${ }^{[37,38]}$. Polycystic ovaries were observed in $45.3 \%$ women with PCOS and increased ovarian volume was observed 
in $27.60 \%$. The significance was also confirmed in the multivariate logistic regression analysis where it stands as risk in PCOS. We also found that the unilateral antral follicular count and ovarian volume of the right ovary was marginally higher than that of the left in women with PCOS.

All the PCOS women who were obese had an increased waist circumference and elevated insulin levels. Waist circumference above the normal range in a woman worsens the symptoms of PCOS. Thus, waist circumference may perhaps be a better indicator than BMI. Of the gynaecological variables, the endometrial thickness, bilateral ovarian volume and bilateral antral follicular count were significantly higher in PCOS women. Even though we found an increase in BMI in PCOS women compared to controls, when trying to ascertain an independent risk factor for PCOS, we found that BMI was not a risk factor and pre-prandial glucose was found to be a protective factor for PCOS. Hence, BMI and pre-prandial glucose should not be considered as major contributing factors in the development of PCOS. We found that elevated testosterone levels lead to 0.11 odds times of risk of developing PCOS. Increased unilateral right and left ovarian volume have 0.20 and 0.15 odds times of risk and increased antral follicular count contribute to 0.27 -odds time risk of developing PCOS in the studied population.

PCOS continues to be of major women's health concern worldwide. It is of importance to obtain a relationship of the various clinical, biochemical, hormonal and gynaecological parameters as risk factors for PCOS. Our study indicates an increase in BMI and waist/ hip ratio may worsen the symptoms of PCOS. Along with BMI, measurement of waist/hip ratio also should be considered. Increased LH/FSH ratio, testosterone, increased pre-prandial glucose levels, ovarian volume and antral follicular count are considered significant risk factors for developing PCOS. The reasons for this observation are yet to be ascertained. As testosterone increases, an increase in LH/FSH ratio, ovarian volume and antral follicular count was observed. These parameters lead to disturbances in the interlinked hormonal and biochemical pathways contributing to PCOS. The features/parameters presented in this study can be utilized for screening purposes to ascertain the susceptibility of women to PCOS. Also, the ethnic and geographical differences observed in the occurrence of PCOS warrant that the screening reaches the maximum number of women in the susceptible age group. One limitation of the study is the sample size, which included a small group of participants. Future research should focus on early detection of the predisposing risk factors in PCOS development, including long-term studies with the goal of modifying environmental factors so that risk may be significantly reduced. The use of Systems Biology approaches in analysing biochemical networks will enable us to better comprehend the multisystem cross-talk underlying the etiology of PCOS.

\section{Acknowledgements:}

We acknowledge funding from the Department of Biotechnology (DBT), Government of India (Project Ref. No. BT/PR14090/GBD/27/275/2010).

\section{Conflict of interest:}

The authors report no conflicts of interest. The authors alone are responsible for the content and writing of the paper.

\section{Financial support:}

None.

\section{REFERENCES}

1. Dasgupta S, Reddy BM. Present status of understanding on the genetic etiology of polycystic ovary syndrome. J Postgrad Med 2008;54:115-25.

2. March WA, Moore VM, Willson KJ, Phillips IWD, Norman RJ, Davies MJ, et al. The prevalence of polycystic ovary syndrome in a community sample assessed under contrasting diagnostic criteria. Hum Reprod 2009;25:544-51.

3. Nidhi R, Padmalatha V, Nagarathna R, Amritanshu R. Prevalence of polycystic ovary syndrome in Indian adolescents. J Pediatr Adolesc Gynecol 2011;24:223-7.

4. Rotterdam ESHRE/ASRM-Sponsored PCOS consensus workshop group. Revised 2003 consensus on diagnostic criteria and long-term health risks related to polycystic ovary syndrome (PCOS). Hum Reprod 2004;19:41-7.

5. Azziz R, Carmina E, Dewailly D, Diamanti-Kandarakis E, Escobar-Morreale HF, Futterweit W, et al. The Androgen Excess and PCOS Society criteria for the polycystic ovary syndrome: the complete task force report. Fertil Steril 2009;91:456-88.

6. Buggs C, Rosenfield RL. Polycystic ovary syndrome in adolescence. Endocrinol Metab Clin North Am 2005;34:677-705.

7. Summer AE, Cowie CC. Ethnic differences in the ability of triglyceride levels to identify insulin resistance. Atherosclerosis 2008;196:696-703.

8. Folsom AR, Kushi LH, Anderson KE, Mink PJ, Olson JE, Hong CP, et al. Associations of general and abdominal obesity with multiple health outcomes in older women: the Iowa Women's Health Study. Arch Intern Med 2000;160:2117-28.

9. Taponen S, Martikainen H, Järvelin MR, Sovio U, Laitinen J, Pouta A, et al. Metabolic cardiovascular disease risk factors 
in women with self-reported symptoms of oligomenorrhea and/or hirsutism: Northern Finland Birth Cohort 1966 Study. J Clin Endocrinol Metab 2004;89:2114-8.

10. Geloneze B, Repetto EM, Geloneze SR, Tambascia MA, Ermetice $\mathrm{MN}$. The threshold value for insulin resistance (HOMA-IR) in an admixture population. IR in the Brazilian metabolic syndrome study. Diabetes Res Clin Pract 2006;72:219-20.

11. Carmina E, Orio F, Palomba S, Longo RA, Cascella T, Colao A, et al. Endothelial dysfunction in PCOS: role of obesity and adipose hormones. Am J Med 2006;119:356.e1-6.

12. Targher G, Solagna E, Tosi F, Castello R, Spiazzi G, Zoppini $\mathrm{G}$, et al. Abnormal serum alanine aminotransferase levels are associated with impaired insulin sensitivity in young women with polycystic ovary syndrome. J Endocrinol Invest 2009;32:695-700.

13. Eden JA, Warren P. A review of 1019 consecutive cases of polycystic ovary syndrome demonstrated by ultrasound. Australas Radiol 1999;43:41-6.

14. De Vries L, Karasik A, Landau Z, Phillip M, Kiviti S, GoldbergStern H. Endocrine Effects of Valproate in Adolescent Girls with Epilepsy. Epilepsia 2007;48:470-7.

15. Lim SS, Davies MJ, Norman RJ, Moran LJ. Overweight, obesity and central obesity in women with polycystic ovary syndrome: a systematic review and meta-analysis. Hum Reprod Update 2012;18:618-37.

16. Vrbikova J, Bendlova B, Hill M, Vankova M, Vondra K, Starka L. Insulin sensitivity and -cell function in women with polycystic ovary syndrome. Diabetes Care 2002;25:1217-22.

17. Taponen S, Martikainen H, Järvelin MR, Laitinen J, Pouta A, Hartikainen AL, et al. Hormonal profile of women with self-reported symptoms of oligomenorrhea and/or hirsutism: Northern Finland birth cohort 1966 study. J Clin Endocrinol Metab 2003;88:141-7.

18. Alili A, Idrizi E. Clinical and biochemical characteristics in women with polycystic ovary syndrome in the region of Pollog, Republic of Macedonia. Eur Sci J 2014;10:179-85.

19. Yousouf R, Khan M, Kounsar Z, Ahangar S. Lone WA. Polycystic ovary syndrome: clinical correlation with biochemical status. Surg Sci 2012;03:245-8.

20. Najem F, Elmehdawi R, Swalem. Clinical and biochemical characteristics of polycystic ovary syndrome in BenghaziLibya: a retrospective study. Libyan J Med 2008;3:71-4.

21. Taylor AE. Determinants of abnormal gonadotropin secretion in clinically defined women with polycystic ovary syndrome. J Clin Endocrinol Metab 1997;82:2248-56.

22. Lasley BL, Wang CF, Yen SS. The effects of estrogen and progesterone on the functional capacity of the gonadotrophs. J Clin Endocrinol Metab 1975;41:820-26.

23. Norman AW, Mizwicki MT, Norman DPG. Steroid-hormone rapid actions, membrane receptors and a conformational ensemble model. Nat Rev Drug Discov 2004;3:27-41.
24. Burt Solorzano CM, McCartney CR, Blank SK, Knudsen KL, Marshall JC. Hyperandrogenaemia in adolescent girls: origins of abnormal gonadotropin-releasing hormone secretion. BJOG 2009;117:143-9.

25. Mauvais-Jarvis P. Regulation of androgen receptor and 5 alpha-reductase in the skin of normal and hirsute women. Clin Endocrinol Metab 1986;15:307-17.

26. Andersson S, Bischop RW, Russell DW. Expression, cloning and regulation of steroid $5 \alpha$-reductase, an enzyme essential for male sexual differentiation. J Biol Chem 1989;264:16249-55.

27. Andersson S, Berman DM, Jenkins EP, Russell DW. Deletion of steroid $5 \alpha$-reductase 2 gene in male pseudohermaphroditism. Nature 1991;354:159-61.

28. Harris G, Azzolina B, Baginsky W, Cimis G, Rasmusson GH, Tolman RL, et al. Identification and selective inhibition of an isozyme of steroid $5 \alpha$-reductase in human scalp. Proc Natl Acad Sci 1992;89:10787-91.

29. Mowszowicz I, Melanitou E, Doukani A, Wright F, Kuttenn F, Mauvais-Jarvis P. Androgen binding capacity and 5[alpha]reductase activity in pubic skin fibroblasts from hirsute patients. J Clin Endocrinol Metab 1983;56:1209-13.

30. Lobo RA, Goebelsmann U, Horton R. Evidence for the importance of peripheral tissue events in the development of hirsutism in polycystic ovary syndrome. J Clin Endocrinol Metab 1983;57:393-7.

31. Loverro G, Vicino M, Lorusso F, Vimercati A, Greco P, Selvaggi L. Polycystic ovary syndrome: relationship between insulin sensitivity, sex hormone levels and ovarian stromal blood flow Gynecol Endocrinol 2001;15:142-9.

32. Buyalos RP, Geffner ME, Bersch N, Judd HL, Watanabe RM, Bergman RN, et al. Insulin and insulin like growth factor-1 responsiveness in PCOS. Fertil Steril 1992;57:796-803.

33. Turhan NO, Toppare MF, Seckin NC, Dilmen G. The predictive power of endocrine test for the diagnosis of polycystic ovaries in women with oligomenorrhea. Gynecol Obstet Invest 1999;48:183-6.

34. Chen J, Shen S, Tan Y, Xia D, Xia Y, Cao Y, et al. The correlation of aromatase activity and obesity in women with or without polycystic ovary syndrome. J Ovarian Res 2015;8:11.

35. Guzick DS. Polycystic Ovary Syndrome. Obstet Gynecol 2004;103:181-93.

36. Tsilchorozidou T, Overton C, Conway GS. The pathophysiology of polycystic ovary syndrome. Clin Endocrinol 2004;60:1-17.

37. Schüring AN, Schulte N, Sonntag B, Kiesel L. Androgens and insulin - two key players in polycystic ovary syndrome. Gynakol Geburtshilfliche Rundsch 2008;48:9-15.

38. Pasquali R, Stener-Victorin E, Yildiz BO, Duleba AJ, Hoeger K, Mason $\mathrm{H}$, et al. PCOS Forum: research in polycystic ovary syndrome today and tomorrow. Clin Endocrinol 2011;74:424-33. 